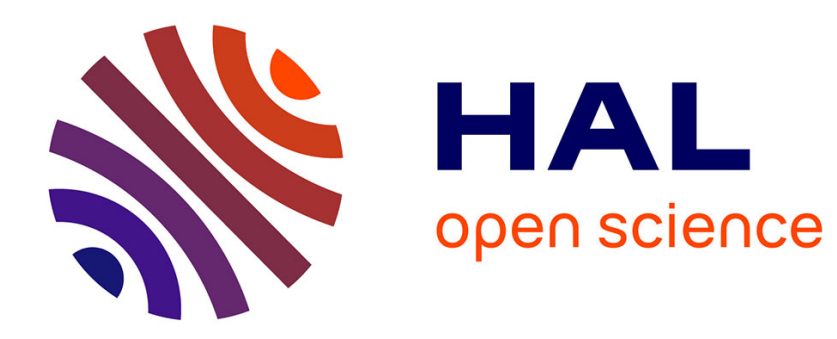

\title{
Sur un spectroscope à grande dispersion
}

A. Cornu

\section{To cite this version:}

A. Cornu. Sur un spectroscope à grande dispersion. J. Phys. Theor. Appl., 1883, 2 (1), pp.53-57. 10.1051/jphystap:01883002005300 . jpa-00238164

\section{HAL Id: jpa-00238164 https://hal.science/jpa-00238164}

Submitted on 1 Jan 1883

HAL is a multi-disciplinary open access archive for the deposit and dissemination of scientific research documents, whether they are published or not. The documents may come from teaching and research institutions in France or abroad, or from public or private research centers.
L'archive ouverte pluridisciplinaire HAL, est destinée au dépôt et à la diffusion de documents scientifiques de niveau recherche, publiés ou non, émanant des établissements d'enseignement et de recherche français ou étrangers, des laboratoires publics ou privés. 


\section{SUR UN SPEGTROSGOPE A GRANDE DISPERSION;}

Par M. A. CORNU.

L'appareil que je vais décrire (1) est un spectroscope à grande dispersion dont l'originalité consiste en ce qu'il ne comprend qu'une seule lunette et qu'un seul prisme.

L'objectif $\mathrm{G}$, achromatique ( $f i g . \mathrm{I})$, a $\mathrm{I}^{\mathrm{m}}, 20$ de distance focale ; la partie utile de son ouverture n'est guère que de $o^{\mathrm{m}}, \mathrm{o} 3 \check{\text { j }}$; il forme à la fois collimateur et lunette; à cet effet, un peu au-dessus de l'axe principal supposé horizontal, se trouve un petit prisme à ré-

Fig. I.

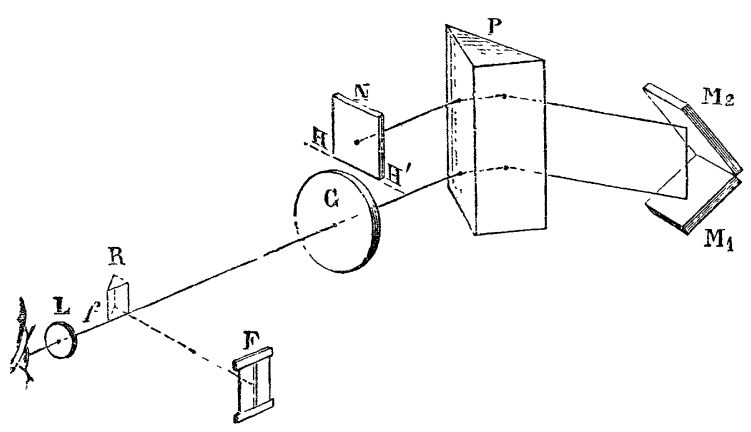

flexion totale $\mathrm{R}$, distant d'environ $\mathrm{o}^{\mathrm{m}}, 9^{5}$ de l'objectif destiné à réfléchir, suivant un axe secondaire, légèrement incliné à l'axe principal, la lumière émise par une fente verticale $\mathbf{F}$ placée au foyer principal de l'objectif. On supposera, pour simplifier l'indication de la marche des rayons, que la source de lumière est monochromatique, ce qui est d'ailleurs le cas réel lorsqu'on règle l'appareil avec la lumière d'un brûleur à gaz contenant une perle de sel marin fondu. Le faisceau émergent, formé de rayons parallèles, tombe sur le prisme $\mathbf{P}$ à arête verticale et se réfracte une promière fois; le faisceau réfracté est reçu sur un miroir à

(') Construit dans les ateliers de M. J. Duboscq.

J. de Phys., $2^{\text {e }}$ série, t. 1I. (Février 1883. ) 
$4{ }^{\circ}, M_{1}$, argenté extérieurement, qui le réfléchit verticalement vers le haut, puis sur un second miroir $\mathbf{M}_{2}$, à angle droit du premier, qui le ramène horizontalement et le fait passer une deuxième fois à travers le prisme, mais à un niveau supérieur : là, le faisceau subit, sur un troisième miroir $\mathrm{N}$, une réflexion presque normale qui lui fait reprendre à peu près le même chemin et le ramène à l'objectif, après quatre réfractions. On règle l'inclinaison du troisième miroir (mobile autour d'un axe horizontal $\mathbf{H H}^{\prime}$ ), de façon que le faisceau de retour soit symétrique du faisceau émergent par rapport à l'axe principal et vienne former en $f$, un peu au-dessous et en arrière du prisme à réflexion totale $R$, l'image de la fente $F$ : un oculaire convenable $\mathrm{L}$ permet d'observer cette image.

Une disposition très simple oriente le prisme de manière

Fig. 2.

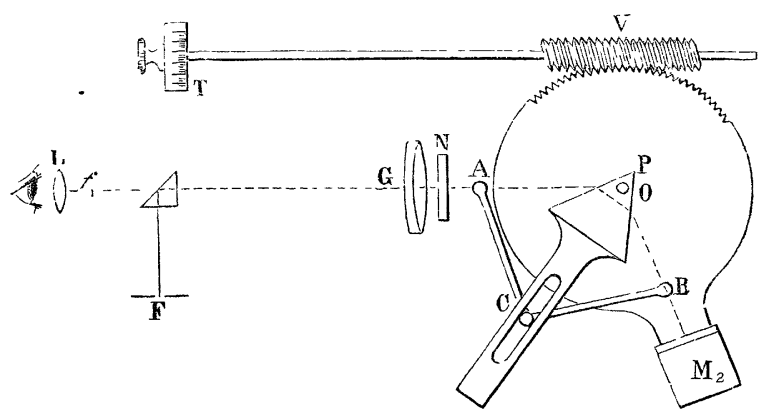

qu'il soit toujours, et pour tous les rayons ramenés dans l'axe de la lunette, au minimum de déviation : à cet effet, le prisme $\mathrm{P}$ et le système des deux miroirs rectangulaires $\mathbf{M}_{1}, \mathbf{M}_{2}(f i g \cdot 2)$ sont montés sur deux alidades indépendantes, $\mathrm{OA}, \mathrm{OB}$, tournant autour du même centre $\mathrm{O}$; le mouvement simultané des alidades est réglé par un quadrilatère articulé AOBC, de façon que le plan bissecteur OC du prisme fasse toujours des angles égaux avec l'axe principal AO de la lunette et le plan de réflexion OR des miroirs, le point A étant fixe. Il suffit, pour cela, d'engager la cheville $\mathrm{C}$ d'articulation des tiges AC, BC dans une fente longitudinale de l'alidade du prisme.

L'observateur règle d'abord les deux miroirs rectangulaires en 
observant les images réfléchies de la prunelle de son oeil, qui doivent se réunir en une seule, coupée en deux moitiés égales par l'arête commune des deux miroirs; il règle ensuite, comme on l'a vu plus haut, l'inclinaison du troisième miroir, de manière à amener en $f$ l'image de la fente. Il est bon d'ôter le prisme d'abord et d'opérer sur les images seulement réfléchies; puis on le remet en place et l'on rectifie. L'emploi d'un faisceau ̇ntense de lumière solaire, qui rend visible la marche des rayons, facilite beaucoup la première approximation : on achève avec la lampe monochromatique.

Lorsque l'appareil est réglé, il suffit de déplacer l'alidade $\mathrm{OB}$ des miroirs rectangulaires, pour faire défiler dans le champ de la lunette la succession des raies spectrales disposées, pour toutes les réfrangibilités, suivant la loi de dispersion que donneraient les quatre prismes placés constamment au minimum de déviation.

On peut faire des mesures micrométriques non seulement avec un micromètre à fil placé à l'oculaire, mais en utilisant le déplacement de l'alidade $\mathrm{OB}$, produit par une vis sans fin $\mathrm{V}$, qui engrène sur le pourtour denté de l'alidade liée à un plateau circulaire. Un tambour divisé $\mathrm{T}$ (qu'on pourrait aisément remplacer par le dispositif de M. Thollon pour l'enregistrement graphique des raies), monté sur la tige de cette vis, se trouve placé près de l'oculaire el permet de mesurer les centièmes de tour et d'apprécier les millièmes.

J'avais improvisé cet appareil, il y a une douzaine d'années, avec un objectif à long foyer et des fragments de glace de SaintGobain; plusieurs savants l'ont vu fonctionner, en 187 r et 1872 , dans mon laboratoire de l'École Polytechnique; j'avais obtenu ainsi, à peu de frais, un long prisme à sulfure de carbone et les trois miroirs argentés décrits ci-dessus. La construction définitive de ce spectroscope, abandonnée et reprise plusieurs fois pour différents motifs, perd beaucoup aujourd'hui de l'actualité qu'elle aurait eue à l'époque où l'appareil a été imaginé. Toutefois il ne le cède en rien, comme simplicité, à la plupart des dispositifs usités depuis et offre même l'avantage, sur beaucoup d'entre eux, de mettre sous la main de l'observateur toutes les pièces à manœuvrer pendant l'observation : le tambour T divisé qu'on manœuvre de la main gauche, la fente $\mathbf{F}$ et son tirage qu'on règle de la main droite, ainsi que la source de lumière. 
Le prisme le plus convenable à employer est un prisme à liquide rempli de sulfure de carbone, qu'il faut soustraire aux variations de température: les prismes de flint sont rarement assez homogènes pour donner de bonnes images. Un prisme de quartz dont le plan bissecteur serait exactement perpendiculaire à l'axe optique pourrait servir spécialement pour la spectrophotographie : le retour des rayons compenserait la double réfraction circulaire.

La principale difficulté réside dans la perfection des surfaces réfringentes et réfléchissantes : il est nécessaire de couper les morceaux à employer dans des glaces beaucoup plus grandes, pour éviter les imperfections des bords. C'est ce qui explique les mécomptes que nous avons éprouvés avec M. Duboscq lorsque nous avons, la première fois, remplacé les glaces du commerce par des glaces travaillées avec soin; les images étaient moins bonnes que celles obtenues avec des surfaces ordinaires : le mieux paraissait l'ennemi du bien. L'exploration optique de l'ensemble des sur- faces avec une lumière monochromatique a montré que, comme toujours, ce sont les bords des surfaces qui sont irréguliers. Dans mes essais faits avec des fragments de glace ordinaire, j'avais donné aux trois miroirs la forme carrée, de façon à les orienter différemment dans leur plan et à chercher méthodiquement la compensation mutuelle de toutes les aberrations. Cette compensation avait fort bien réussi, et je conseillerais de la tenter dans des cas analogues.

On peut répéter avec le spectroscope toutes les observations qui exigent une grande dispersion : je me bornerai à citer une expérience qui donnera une idée précise de la puissance de l'appareil.

Si l'on remplace le troisième miroir $\mathbf{N}$ argenté extérieurement par une glace argentée intérieurement, mais à faces bien parallèles, le spectre continu d'une lumière intense est sillonné de franges serrées. Ces franges sont dues à l'interférence des ondes réfléchies aux deux surfaces; c'est donc l'expérience de Fizeau et Foucaulı sur l'interférence à grande différence de marche. Le spectroscope est assez dispersif pour permettre de compter i 7 à i 8 franges sombres dans l'intervalle compris entre les deux raies $\mathrm{D}$ du spectre solaire, avec un miroir formé d'une glace d'environ $3^{\mathrm{mm}} \mathrm{d}^{\text {'épaisseur. }}$ 
Le calcul montre qu'en effet c'est bien là le résultat qu'on doit trouver ( $\left.{ }^{1}\right)$.

L'appareil se prête en outre très bien à la spectrophotographie; mais il est bon d'éliminer alors la lumière diffusée par les points brillants de l'objectif, à l'aide de très petits morceaux de papier noir collés sur l'objectif à l'intérieur de la lunette.

Pour l'observation optique, il n'est pas besoin de cette précaution; il suffit d'employer l'ingénieux artifice imaginé par M. Fizeau dans ses expériences sur la vitesse de la lumière et qui consiste à cacher sur l'anneau oculaire l'image conjuguée des points brillants de l'objectif par un petit fil métallique fixé à l'œilleton de l'oculaire.

(') Soient $\varphi_{1}$ la différence de phase introduite par l'épaisseur $2 e$ de verre d'indice $n_{1}$ pour la raie $\mathrm{D}_{1} ; \varphi_{2}, n_{2}$ les coefficients analogues pour la raie $\mathrm{D}_{2}$. Le nombre de franges comprises entre ces deux raies sera

$$
\varphi_{1}-\varphi_{2}=2 e\left(\frac{n_{1}}{\lambda_{1}}-\frac{n_{2}}{\lambda_{2}}\right),
$$

ou très approximativement, si la différence $\lambda_{1}-\lambda_{2}$ est assez petite pour être considérée comme une différentielle $d \lambda$,

$$
\varphi_{1}-\varphi_{2}=2 e \frac{d}{d \lambda}\left(\frac{n}{\lambda}\right) d \lambda .
$$

Pour un crown-glass de même nature, j'ai obtenu comme indices

$$
\begin{array}{ll}
\text { Raie C.... } n^{\prime}=1,514498 & \lambda^{\prime}=0^{\mathrm{mm}}, 0006562 \mathrm{x} \\
\text { Raie } \mathrm{D}_{2} \ldots n^{\prime \prime}=\mathrm{I}_{1}, 5_{1} \mathrm{I}_{107} & \lambda^{\prime \prime}=\mathrm{o}^{\mathrm{mm}}, 00058889
\end{array}
$$

Déterminant les coefficients de la fonction approchée,

$$
n=a+\frac{b}{\lambda^{2}} \quad\left(a=1,50372, \quad b=0,000000004647^{\prime} 3\right),
$$

d'où l'on conclut

$$
\frac{d}{d \lambda}\left(\begin{array}{l}
n \\
\lambda
\end{array}\right)=-\frac{a}{\lambda^{2}}-\frac{3 b}{\lambda^{4}} .
$$

Substituant la valeur $\lambda$ qui correspond à la raie $\mathrm{D}_{2}$, on obtient le nombre 4452 roo. Or, la valeur $d \lambda$ est la différence $\lambda_{2}-\lambda_{1}$.

$$
\begin{array}{ll}
\text { Raie } D_{1} \ldots & \lambda_{1}=0^{\mathrm{mm}}, 00058948 \\
\text { Raie } D_{2} \ldots & \lambda_{2}=0^{\mathrm{mm}}, 00058889
\end{array}
$$

Substituant dans la valeur de $\varphi_{1}-\varphi_{2}$, on trouve 15,8 franges, approximation en rapport avec les petites incertitudes sur les constantes de la dispersion et sur l'épaisseur de la glace qui n'a pu ètre mesurée qu'un peu grossièrement. 